\title{
Reconstruction performance studies for liquid argon time projection chambers with two and three readout planes
}

\author{
D. Brailsford ${ }^{1}$, M. B. Brunetti ${ }^{2}$, S. Alonso Monsalve ${ }^{3}$, A. Blake ${ }^{1}$, A. Chappell ${ }^{2}$, J. \\ Marshall $^{2}$, L. H. Whitehead ${ }^{4}$, and E. Worcester ${ }^{5}$ \\ ${ }^{1}$ Lancaster University, Bailrigg, Lancaster LA1 4YB, United Kingdom \\ ${ }^{2}$ University of Warwick, Coventry CV4 7AL, United Kingdom \\ ${ }^{3}$ ETH Zurich, Institute for Particle Physics, Zurich, Switzerland \\ ${ }^{4}$ University of Cambridge, JJ Thomson Avenue, Cambridge CB3 OHE, United Kingdom \\ ${ }^{5}$ Brookhaven National Laboratory, Upton, NY 11973, USA
}

\section{Introduction}

One of the original questions in defining the design of the Vertical Drift (VD) Liquid Argon Time Projection Chamber (LArTPC) was whether to adopt a configuration with three or two readout planes. While the former is a well-established technology and has since been identified as the reference configuration, initial studies, carried out in the context of event reconstruction and described in this document, indicate that the latter option would have potentially lower performance. However, as it would present significant costsaving advantages, DUNE is continuing to develop 2-view techniques in parallel to other reconstruction development. At the time of writing, the VD detector software is still under development and not available for use. Therefore, the well-established geometry and software for the Horizontal Drift (HD) detector has been used as a temporary measure.

\section{Using the HD simulation and reconstruction for VD perfor- mance studies}

Both horizontal and vertical drift detectors work under the same general principle, where each readout plane observes a $2 \mathrm{D}$ projection of a neutrino interaction, commonly referred to as a 'view'. This is true despite any of the proposed differences in the readout systems and drift orientation.

One of the reconstruction's tasks is to create full 3D representations of neutrino interactions in the detector. This is achieved by creating clusters of hits in each view and then matching those clusters between the readout planes, correlating timing or charge information, to create 3D particles. This procedure is also the same for both detector types.

Because of this equivalence, observed changes in reconstruction performance when varying common features (two-views vs three-views, readout orientation and pitch, etc.) in one detector should reflect the performance changes in the other detector. This detector equivalence has been previously exploited for ProtoDUNE-DP (a two-view, strip-based LArTPC) where the Pandora [5, 6] reconstruction, designed in the scope of a three-view, wire-based LArTPC, successfully reconstructed ProtoDUNE-DP Monte Carlo (MC) without modification.

Considering the above, it is possible to utilise the well-established simulation and reconstruction of the horizontal drift far detector to study how design choices in the VD detector could impact its performance. Therefore, all of the following optimisation studies revolve around the ' $1 \times 2 \times 6$ ' HD geometry and its accompanying simulation and reconstruction, as used extensively in the DUNE Technical Design Report (TDR) [1].

This manuscript has been authored by Fermi Research Alliance, LLC under Contract No. DE-AC02-07CH11359 with the U.S. Department of Energy, Office of Science, Office of High Energy Physics. 


\section{Two-view and three-view event reconstruction with Pandora}

Pandora is a pattern recognition suite that employs a multi-algorithm approach to LArTPC neutrino reconstruction. It provides algorithms for all of the $2 \mathrm{D}, 2 \mathrm{D} \rightarrow 3 \mathrm{D}$, and $3 \mathrm{D}$ reconstruction stages outlined in section 2 .

The Pandora reconstruction chains for the case where three readout planes are available is well-established, and validated in detectors such as MicroBooNE[5] and ProtoDUNE-SP [2], while the reconstruction chain for detectors with two readout planes has been deployed for the first time more recently.

The $2 \mathrm{D}$ reconstruction stage creates clusters from observed hits in each of the $2 \mathrm{D}$ views, obtained from the waveforms in a specific readout plane, having coordinates derived from the common drift time and wire/strip position. This part of the reconstruction operates on each view in isolation, meaning that the number of available views does not impact the $2 \mathrm{D}$ reconstruction performance.

The main divergence between a two-view and three-view reconstruction is expected to be the $2 \mathrm{D} \rightarrow 3 \mathrm{D}$ matching procedure, which aims at matching together clusters that correspond to the same particle across different views in order to reconstruct 3D trajectories. In brief, common timing information associated with the $2 \mathrm{D}$ clusters is exploited to match the clusters together, but the specific mechanism largely depends on how many views are available to the reconstruction.

When three views are available to the $2 \mathrm{D} \rightarrow 3 \mathrm{D}$ matching, clusters in two of the views can be combined using the common timing information and geometric rotations to infer locations along the cluster in the third view. These inferred locations can be compared to the actual locations along the third-view cluster, providing a powerful geometric method for matching $2 \mathrm{D}$ clusters across the readout planes. The $2 \mathrm{D} \rightarrow 3 \mathrm{D}$ matching stage also uses the third view to recover cases where one of the views is poor, and to correct $2 \mathrm{D}$ pattern recognition mistakes if one of the views is in disagreement with the others, especially for complex topologies. For example, the view redundancy allows recovery of a kink in one of the particle trajectories that manifests itself at the $2 \mathrm{D}$ level only in one of the views.

When only two views are available, such location inference (as with three-view $2 \mathrm{D} \rightarrow 3 \mathrm{D}$ matching) is not possible and the $2 \mathrm{D} \rightarrow 3 \mathrm{D}$ matching procedure can only compare the timing information associated with the end points of the two available clusters. This two-view geometric matching method is less capable of resolving matching ambiguities than its three-view counterpart.

A new two-view matching method was recently developed that exploits calorimetric and timing information simultaneously to help over-constrain the $2 \mathrm{D} \rightarrow 3 \mathrm{D}$ matching. This calorimetric method compares and correlates the energy depositions as a function of time along the two clusters, where a high degree of correlation implies a strong match.

The reconstruction for both three-view and two-view detectors is under active development. The reconstruction performance is expected to increase for both configurations and eventually start to approach the intrinsic physical limitations of both options.

\section{Two-view and three-view performance studies}

Two MC simulation studies have been undertaken using two iterations of the $1 \times 2 \times 6$ horizontal drift geometry: the reference geometry, with two induction planes with wires forming $35.7^{\circ}$ with the vertical and a collection plane with vertical wires, and an alternative geometry where the two induction planes form a $90^{\circ}$ angle with each other and a $45^{\circ}$ angle with the vertical. To test how the number of available views impacts the reconstruction, the $\mathrm{MC}$ samples were processed through three separate configurations of the Pandora reconstruction:

1. The standard three-view reconstruction and using input hits from all three views;

2. Running the standard three-view reconstruction and only using inputs from the two induction views, essentially mimicking a two-view detector. Because only two-views are available to the reconstruction, the $2 \mathrm{D} \rightarrow 3 \mathrm{D}$ matching resorts to the two-view geometric method, comparing cluster end points using timing information; 


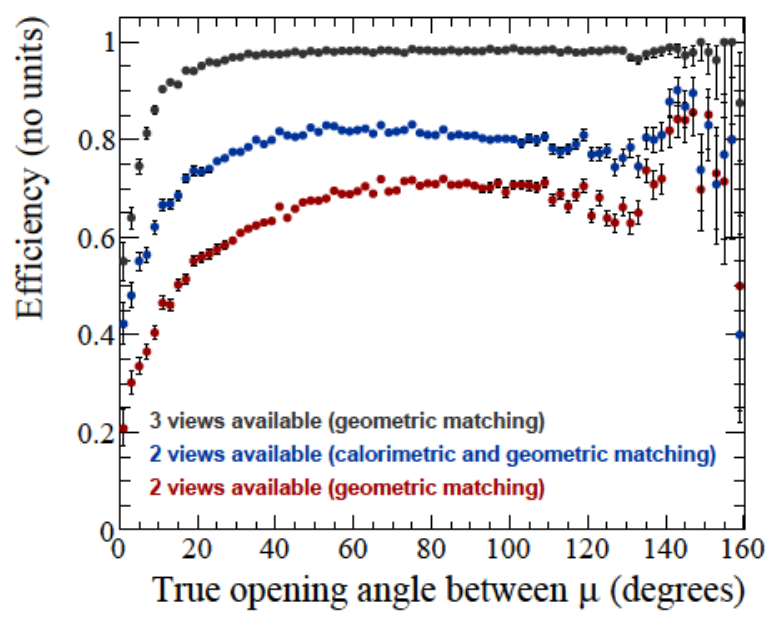

((a))

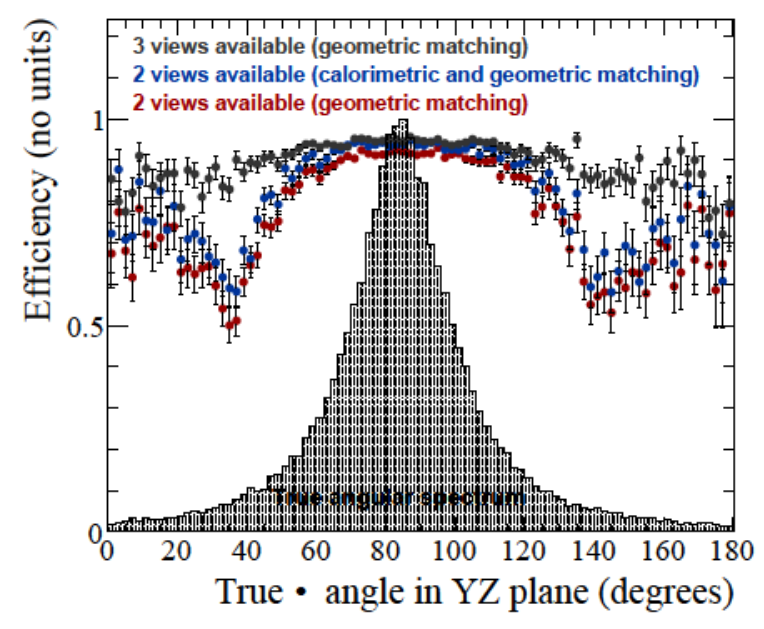

((b))

Figure 1: Reconstruction efficiency as a function the angle in the horizontal plane between the two muons in a MC di-muon particle gun sample (a) and as a function of the angle of the muon in the anode (YZ) plane for a CC $\nu_{\mu}$ sample, overlaid with the angular spectrum of generated muons (b). The different coloured points represent different reconstruction configurations, as indicated in the legend.

3. Same configuration as in 2 and also adding the novel two-view calorimetric $2 \mathrm{D} \rightarrow 3 \mathrm{D}$ matching.

The reconstruction performance for the three configurations described above was studied for two different samples, a di-muon particle gun sample and a CC $\nu_{\mu}$ sample simulated using GENIE. In both cases an event is deemed reconstructed if all primary muons match at least one reconstructed particle. The contributing views of the reconstructed particle must also all match to the same true muon. The reconstruction efficiency is then defined as the fraction of reconstructed events according to the above definition.

\subsection{Di-muon particle gun study}

The di-muon sample was generated in the HD $1 \times 2 \times 645^{\circ}$ geometry with both muons originating from a fixed starting point near the cathode of one of the TPCs, a flat angular spectrum between $-70^{\circ}$ and $+70^{\circ}$ for both $\theta_{x z}$ and $\theta_{y z}$, and a flat momentum distribution between $0.2 \mathrm{GeV}$ and $2.5 \mathrm{GeV}$. The $45^{\circ}$ geometry was used as the two induction planes subtend an angle that was similar to one of the candidate two-view VD detector designs at the time the studies were first undertaken.

Fig. 1(a) shows the reconstruction efficiency as a function of the opening angle between the two muons. In all configurations, the fall in efficiency at small opening angles is due to the muons being in close proximity, and reconstructed as a single particle, or one of the two being attributed most of the hits resulting in a poorly reconstructed second muon. The fall and rise in efficiency for a two-view configuration at $145^{\circ}$ is due to the fact that, in a fraction of events, both muons are parallel to the readout-wires in one of the views, yielding worse signal and therefore resulting in reduced acceptance. When a third non-parallel view is present, it is exploited to recover the performance for such topologies.

The integrated reconstruction efficiency quickly approaches $100 \%$ for the three-view configuration (black), and it falls by a few tens of percent when dropping the input from one of the views (red). When adding the calorimetric $2 \mathrm{D} \rightarrow 3 \mathrm{D}$ matching, the loss is partially recovered (blue). 


\subsection{GENIE-simulated Charged-Current (CC) $\nu_{\mu}$ study}

$\mathrm{CC} \nu_{\mu}$ interactions were simulated in the nominal $1 \times 2 \times 6$ HD geometry, using an identical configuration to that used in the TDRs[1] oscillation analysis. As before, the simulated events were processed through the three alternative reconstruction workflows.

The reconstruction efficiency of the final-state muon for each of the three workflows is shown in Fig. 1(b). The efficiency is shown as a function of the muon's angle in the anode plane $\left(0^{\circ}\right.$ and $90^{\circ}$ are defined as vertical and forward-going respectively). The true angular spectrum of the muons is also included in the figure. As with the di-muon study, there is a small overall drop in reconstruction performance when only two views are available to the reconstruction, as the integrated efficiency goes down from 92.7 to 86.2 , but the performance can be partially recovered to an efficiency of 89.1 with the more advanced $2 \mathrm{D} \rightarrow 3 \mathrm{D}$ matching procedures. However, there are significant differences in performance when the muons are near-parallel to the wire orientations $\left(35.7^{\circ}\right.$ and $\left.144.3^{\circ}\right)$. When only two views are available to the reconstruction, regardless of how advanced the $2 \mathrm{D} \rightarrow 3 \mathrm{D}$ matching procedures are, the efficiency notably lowers to $\sim 55 \%$. When three views are available, the reconstruction performance is largely impervious to the inclination of the muons.

\subsection{Conclusions}

A few observations can be made. Firstly, there are physical topologies that are inherently challenging to reconstruct in a two-view detector. A muon that is near-parallel to one of the readout orientations would leave little usable information in that view, meaning only one reconstructable view would be available, making $3 \mathrm{D}$ reconstruction much more challenging.

Secondly, the choice of readout orientation has little effect on the reconstruction performance provided that three views are available, as inferred from the results of the CC $\nu_{\mu}$ study. This finding has implications for future design choices. We would expect a three-view, strip-based VD detector to have a similar flat efficiency curve to that shown in Fig. 1(b). Likewise, a two-view VD detector would most likely exhibit similar dips in performance when the CC $\nu_{\mu}$ muons are near parallel to the strips. If such a two-view VD detector's views were oriented in a similar way to the induction planes used in Fig. 1(b), then only a small drop in the integrated reconstruction efficiency should be expected. However, if one of the readout views was parallel to the beam direction, the dip in the reconstruction efficiency curve would align with the peak of the angular spectrum and a much more significant overall drop in efficiency would be observed.

With sufficient development time and the deployment of novel tools and techniques, it is possible that lost performance in the two-view reconstruction would partially be recovered, and the reconstruction performance of both options would approach their intrinsic detector limitations.

\section{$5 \quad$ Neutrino flavour tagging using a Convolutional Neural Network $(\mathrm{CNN})$}

A CNN [4] is used to perform the neutrino event classification in the Far Detector (FD) neutrino oscillation analyses [3] at DUNE. The CNN takes images as input, where each image contains the hit charge at a given time for each wire that forms a readout view. Thus, the standard CNN takes three input images, and returns a score that gives the likelihood that an event is either: $\mathrm{CC} \nu_{e}, \mathrm{CC} \nu_{\mu}, \mathrm{CC} \nu_{\tau}$ or NC. Three simple studies using the horizontal drift detector were performed by reducing the number of input images for the CNN:

1. Use only the collection view $\left(90^{\circ}\right.$ to the beam)

2. Use the collection view and the second induction view $\left(90^{\circ}\right.$ and $54^{\circ}$ to the beam)

3. Use the two induction views $\left( \pm 54^{\circ}\right.$ to the beam $)$

The CNN architecture was modified and the network retrained in each of the three cases. The CC $\nu_{e}$ selection efficiencies are shown in Figure 2, and the differences between the three cases are apparent at low energy. 
Taken from vertical drift detector proposal

\begin{tabular}{|lc|c|c|}
\hline Configuration & & $\nu_{e}$ CC & $\nu_{\mu}$ CC \\
& & purity @ efficiency & purity @ efficiency \\
\hline DUNE CVN & $(3$ views $)$ & $0.92 @ 0.89$ & $0.97 @ 0.94$ \\
Collection plane only & $(1$ view $)$ & $0.87 @ 0.81$ & $0.96 @ 0.88$ \\
Two induction planes & $(2$ views $)$ & $0.85 @ 0.85$ & $0.96 @ 0.91$ \\
Collection and one induction plane $(2$ views $)$ & $0.86 @ 0.84$ & $0.96 @ 0.91$ \\
\hline
\end{tabular}

rino energy values. These beam direction, but that

\footnotetext{
- Reminder: Convolution Visual Network (CVN) used as the sole neutrino flavour ID algorithm for the far detector oscillation analysis in the TDR

- CVN assesses reconstructed energy depositions in all available readout views simultaneously

- The CVN was exposed to different combinatorics of readout views to assess performance impact

- The nominal horizontal drift far detector geometry was used for each test

- Neutrino flavour identification performance weakly depends on the number of available views
}

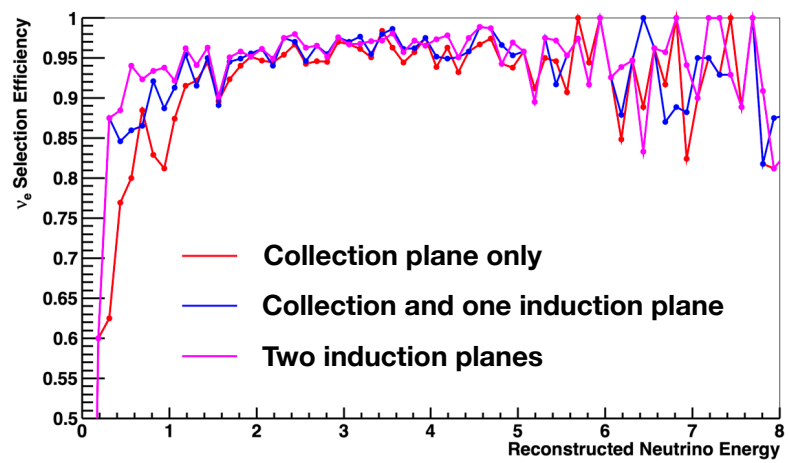

Figure 2: CC $\nu_{e}$ selection efficiency as a function of reconstructed neutrino energy when the neutrino flavourtagging $\mathrm{CNN}$ is exposed to a reduced number of input images.

\section{Summary and outlook}

In this chapter, initial studies to assess performance differences in the reconstruction of a two-view and a three-view detector were presented. While the 3D reconstruction studies using Pandora, performed in the HD $1 \times 2 \times 6$ geometry, at this stage suggest that a two-view VD detector would under-perform with respect to a three-view detector, further development of the reconstruction for the former configuration may reduce the performance difference. In particular, ongoing effort is focused on further developing the calorimetric $2 \mathrm{D} \rightarrow 3 \mathrm{D}$ matching. Conversely, whole-event neutrino flavour tagging, as performed with the CNN, is largely unaffected by the number of available readout planes.

As soon as the reconstruction interface is configured, the studies will migrate to the full VD geometry, although no significant change is expected in the results.

\section{References}

[1] B. Abi et al. Deep Underground Neutrino Experiment (DUNE), Far Detector Technical Design Report, Volume II: DUNE Physics. 2020. arXiv: 2002.03005 [hep-ex].

[2] B. Abi. "First results on ProtoDUNE-SP liquid argon time projection chamber performance from a beam test at the CERN Neutrino Platform". In: Journal of Instrumentation 15.12 (Dec. 2020), P12004P12004. ISSN: 1748-0221. DOI: 10.1088/1748-0221/15/12/p12004. URL: http://dx.doi .org/10. 1088/1748-0221/15/12/P12004.

[3] B. Abi et al. "Long-baseline neutrino oscillation physics potential of the DUNE experiment". In: Eur. Phys. J. C 80.10 (2020), p. 978. DOI: $10.1140 /$ epjc/s10052-020-08456-z. arXiv: 2006.16043 [hep-ex].

[4] B. Abi et al. "Neutrino interaction classification with a convolutional neural network in the DUNE far detector". In: Phys. Rev. D 102.9 (2020), p. 092003. DOI: 10.1103/PhysRevD . 102 . 092003. arXiv: 2006.15052 [physics.ins-det].

[5] R. Acciarri et al. "The Pandora multi-algorithm approach to automated pattern recognition of cosmicray muon and neutrino events in the MicroBooNE detector". In: Eur. Phys. J. C 78.1 (2018), p. 82. DOI: $10.1140 / \mathrm{epjc} / \mathrm{s} 10052-017-5481-6$. arXiv: 1708.03135 [hep-ex].

[6] J.S. Marshall, A. Münnich, and M.A. Thomson. "Performance of Particle Flow Calorimetry at CLIC". In: Nucl. Instrum. Meth. A 700 (2013), pp. 153-162. DOI: 10.1016/ j. nima.2012 .10.038. arXiv: 1209.4039 [physics.ins-det]. 